\title{
Signal integration enhances the dynamic range in neuronal systems
}

\author{
Leonardo L. Gollo, Claudio Mirasso, and Víctor M. Eguíluz \\ IFISC (CSIC-UIB), Instituto de Física Interdisciplinar y Sistemas Complejos, E-07122 Palma de Mallorca, Spain
}

(Received 20 September 2011; revised manuscript received 9 December 2011; published 23 April 2012)

\begin{abstract}
The dynamic range measures the capacity of a system to discriminate the intensity of an external stimulus. Such an ability is fundamental for living beings to survive: to leverage resources and to avoid danger. Consequently, the larger is the dynamic range, the greater is the probability of survival. We investigate how the integration of different input signals affects the dynamic range, and in general the collective behavior of a network of excitable units. By means of numerical simulations and a mean-field approach, we explore the nonequilibrium phase transition in the presence of integration. We show that the firing rate in random and scale-free networks undergoes a discontinuous phase transition depending on both the integration time and the density of integrator units. Moreover, in the presence of external stimuli, we find that a system of excitable integrator units operating in a bistable regime largely enhances its dynamic range.
\end{abstract}

DOI: 10.1103/PhysRevE.85.040902

PACS number(s): 87.19.L-, 05.45.-a, 05.70.Fh, 89.75.Hc

Introduction. A system operating in the vicinity of a critical state can present several advantages. For instance, hair cells of the auditory system poise themselves close to a Hopf bifurcation [1], and in neuronal systems it has been proposed to provide optimal solutions for sensory stimuli detection [2,3], the transmission and storage of information [4,5], and computational capabilities [6]. These results motivated discussions of how the brain can, if it does, operate in a critical state and whether it could be due to self-organization arguments [7] or by evolutionary reasons [8]. Neural systems operating in a critical state also provide an alternative explanation of how the brain integrates the activity of distant regions [4]. In the critical regime, the correlation length diverges and neurons from different areas can effectively share information. Based on these arguments and on experimental evidences [9], it has been suggested that the brain should be tuned around a critical point of a second-order phase transition to efficiently process information [9,10].

Excitable media have been proved to serve as excellent stimulus intensity processors. Their fundamental nonlinear interactions of excitable waves confer a great capacity to compress several decades of stimulus intensity inputs into a single decade of firing rate output [11]. This capability, which has also been proposed to be the main function of neuronal active dendrites [12], is robust for different networks [2,12-14]. In many contexts, such as gene regulatory networks [15], and neuronal [16] and social systems [17], the typical elementary unit dynamics results from the integration of neighbor contributions. In neuroscience, it remains a fundamental open problem to understand how a singular membrane potential output is generated by the convergence of complex spatio-temporal synaptic integration $[11,12,18]$. To accrue for this difficulty, neurons present a myriad of active channels [19], dendritic structures (even within the same neuron type [20]), and temporal integration modes. For example, the efficacy of the presynaptic neurons is largely variable, and neurons might require up to hundreds of excitatory postsynaptic potentials to spike [16].

\footnotetext{
*leonardo@ifisc.uib-csic.es
}

In this Rapid Communication we demonstrate that integration of excitable units is a central element to shape the dynamics of the system: The nonequilibrium phase transition, between the resting and the self-sustained configurations, switches from a continuous second-order to a discontinuous first-order transition. Along with this discontinuity, a historydependent bistable phase emerges. In this phase, the inputoutput response changes and the dynamic range is strikingly enhanced. We show the generality of the result with respect to the network topology, the integration time window, and the number of input signals needed to fire. Moreover, we point out how the presence of a bistable phase changes the paradigm of maximum dynamic range at criticality [2]. Such an optimum regime typically appears in the bistable regime and depends on the past history.

The model. As a simple and influential excitable media, we explore the Kinouchi-Copelli model [2,21] generalized to account for the integration of multiple excitatory inputs. We consider $N$ nodes embedded in sparse (Erdős-Rényi) random and (Barabási-Albert) scale-free networks [22], both with an average degree $K=50$. Each node $i$ represents an excitable unit whose state $s_{i}(t) \in\{0,1,2\}$ indicates whether the unit is in the quiescent state $\left[s_{i}(t)=0\right]$, in the active state $\left[s_{i}(t)=1\right]$, or in the refractory state $\left[s_{i}(t)=2\right]$. The dynamics obeys probabilistic rules with a synchronous update, and $\delta t \equiv 1 \mathrm{~ms}$ is the discrete time step. Every node $i$ at time $t$ updates its state as follows: (a) In the active state $s_{i}(t)=1$, it switches to the refractory state $s_{i}(t+\delta t)=2$; (b) in the refractory state $s_{i}(t)=2$, it returns to the quiescent state $s_{i}(t+\delta t)=0$ with probability $p_{\gamma}=\frac{1}{2}$; and (c) nodes in the quiescent state $s_{i}(t)=0$ become active either (i) by an external driving (or spontaneous activation) with probability $p_{h}=1-\exp (-h \delta t)$ per time step, where $h$ is the rate of a Poisson process, or (ii) by the integration of the contributions received from their active neighbors, with probability $p_{\lambda}$.

In order to model the integration process, we count the number of neighbor contributions $\Lambda_{i}(t)$ received within the time window of width $\tau:(t-\tau, t)$. In the absence of external driving, a node $i$ spikes if $\Lambda_{i}$ reaches at least $\theta$ inputs, i.e., $\Lambda_{i}(t) \geqslant \theta$. Two extreme limits of integration time are of particular interest: the infinite integration time $\tau \rightarrow \infty\left(\tau_{\infty}\right)$, where the integration window takes into account the entire current 

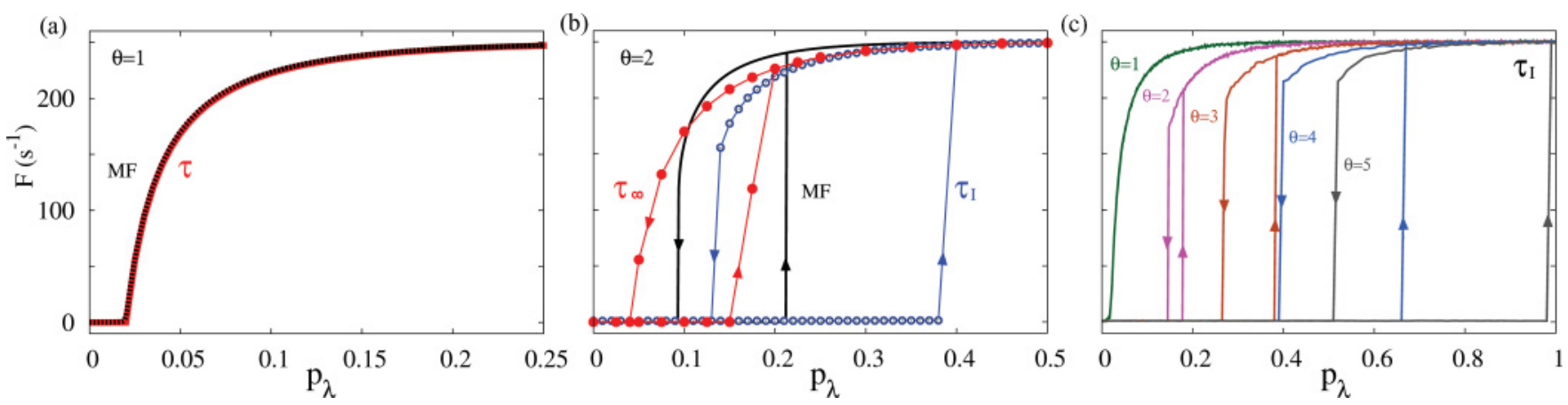

FIG. 1. (Color online) Continuous and discontinuous spontaneous activity $F$ versus $p_{\lambda}$. Mean-field approximation (MF) and numerical results for random networks of (a) nonintegrators $(\theta=1)$, (b) integrators $(\theta=2)$ for both $\tau_{I}$ and $\tau_{\infty}$ integration times with $N=5000$; and (c) integrators with different threshold values, for $\tau_{I}$ and $N=1000$. Other parameter values are $\Delta p_{\lambda}=0.0025$ and $F_{0}=3 \%$.

quiescent history of the node, and a coincidence detection $\tau=1 \mathrm{~ms}\left(\tau_{I}\right)$, where the integration time is limited to $\delta t$.

Continuous versus discontinuous phase transition. In the absence of external driving $(h=0)$, the standard model without integration $(\theta=1)$ leads to a continuous phase transition [2]. The average firing rate $F$, calculated over all nodes and over a large time window (10 s), grows smoothly for increasing coupling strength above the critical value $p_{\lambda}^{c}$ [Fig. 1(a)]. The critical point is determined by the largest eigenvalue of the network adjacency matrix [14]. For a random network [Fig. 1(a)], the critical value is $p_{\lambda}^{c}=K^{-1}$, when the average number of spikes induced by each spike (branching ratio) is one [2]. Conversely, in the presence of integration $(\theta>1)$, the phase transition occurs abruptly, generating a bistable phase with a hysteresis cycle (see the mean-field approach below). We calculated the hysteresis cycles by varying $p_{\lambda}$ upward and downward along the whole range in small steps of $\Delta p_{\lambda}$, activating at each change of $p_{\lambda}$ a small fraction of nodes ( $F_{0}$, from $1 \%$ to $3 \%$ ) to allow the system to escape from the resting configuration. As shown in Fig. 1(b), the change in the nature of the phase transition is observed for any value of the integration time, as well as in the mean-field approximation. The discontinuous phase transition is also robust for any value of $\theta>1$, illustrated in Fig. 1(c) for $\tau_{I}$. It can be also seen from the figure that larger threshold values generate larger hysteresis cycles.

While the previous analysis assumes identical nodes, next we consider heterogeneous populations composed of both integrators $(\theta=2)$ and nonintegrators $(\theta=1)$ nodes. This situation corresponds to the intermediate configuration between integrators, as in Fig. 1(b), and nonintegrators, as in Fig. 1(a). For random and scale-free networks, the minimum density of integrator nodes $(d)$ that yields a discontinuous phase transition depends on the integration time scale $\tau$, as shown in Fig. 2. Although in both cases the density of integrators needed to display a discontinuous phase transition decreases with increasing integration time, the scale-free network requires a lower density of integrators. The integration time is fundamental to bind the collective dynamics together. Coincidence detection restricts the scope of action of the integrator nodes and the network is effectively split in two parts according to the threshold values. For example, in a random network with $\tau_{I}$ and a density of integrators below $80 \%$, the dynamics is dominated by the subgroup of active nonintegrators, leading to a continuous phase transition. In this case of continuous transition, the integrator nodes do not interfere much in the dynamics: The effective connectivity is $K(1-d)$, and the expected critical point for the phase transition is given by $p_{\lambda}^{c} \simeq \frac{1}{K(1-d)}$ (for the left inset panel of Fig. 2: $K=50$, and $d=0.7, p_{\lambda}^{c}=\frac{1}{15}$ ). For larger integration times ( $\tau>\tau_{I}$ ), the discontinuous phase transition (as exemplified by the right inset panel) gradually dominates, and the right side of the transition increases with $\tau$. In this case, the integrator nodes, although spiking less, tend to remain active, furnishing clear influence in the collective dynamics. Therefore, the prevailing dynamics carries the integrators fingerprint given by the discontinuous phase transition.

Dynamic range. So far we have analyzed the behavior of the excitable media in the absence of external stimuli. In the remainder, we are interested in the response of the

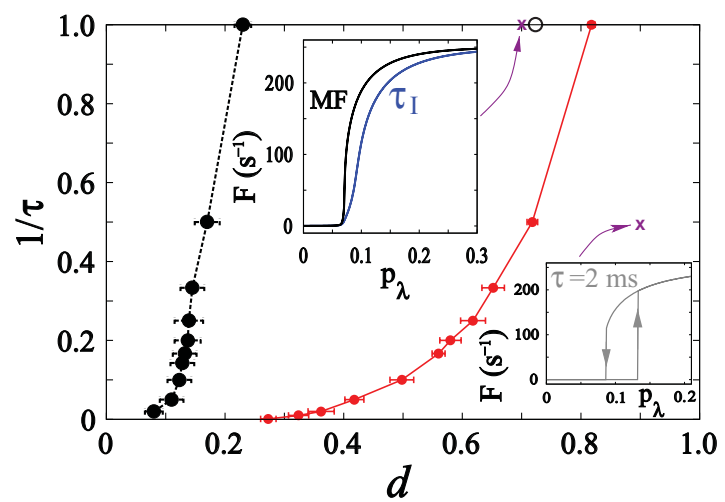

FIG. 2. (Color online) Dependence of the nature of the phase transition order on the integration time $\tau$ for networks $(N=5000)$ composed of a mixture of both integrators (with a density $d$ of $\theta=$ 2 nodes) and nonintegrators. The solid (dashed) line corresponds to the random (scale-free) network for $\Delta p_{\lambda}=0.001$ and $F_{0}=1 \%$. The left-hand side of the curve corresponds to a continuous phase transition whereas the right-hand side corresponds to a discontinuous phase transition. The error bars correspond to the standard deviation over ten trials. The black open symbol depicts the mean-field shift in the order of the phase transition. The left inset panel compares the mean-field approximation with the simulations for the density of integrators $d=70 \%$ and $\tau_{I}$. The right inset panel illustrates a discontinuous phase transition for $\tau=2 \mathrm{~ms}$ and $d=90 \%$. 

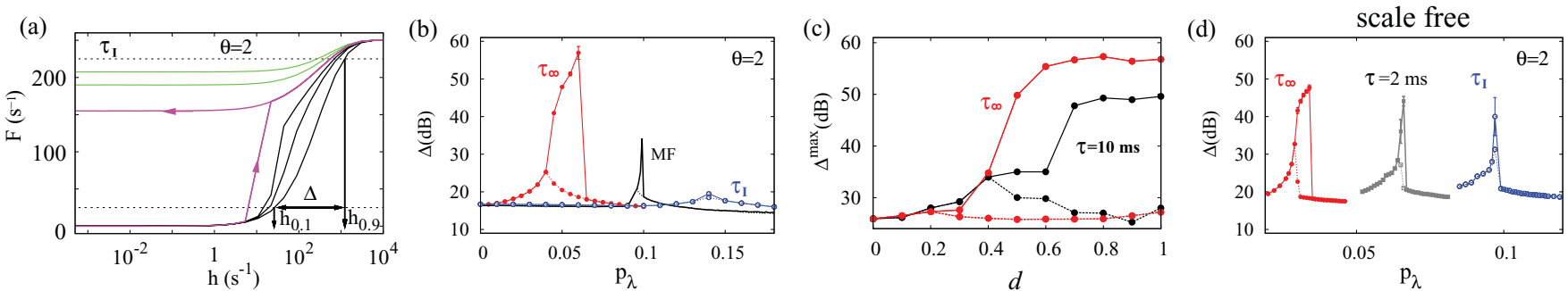

FIG. 3. (Color online) Response curves and dynamic range in networks of integrators $(\theta=2)$ with $N=5000$. (a) Family of response functions for a random network with $\tau_{I}$ and (from right to left) $p_{\lambda}=0,0.1,0.12,0.14,0.16,0.18$. (b) and (d) Dynamic range vs coupling strength for (b) random and (d) scale-free networks. At the bistable region, the dotted line (bottom) stands for initial conditions with a high activity level and the continuous line (top) stands for initial conditions with a low activity level. The error bars correspond to the standard deviation over six trials of two realizations. (c) Dependence of the maximum dynamic range $\Delta^{\max }$ on the density of integrators in random networks.

system as a function of the external driving, considered as a Poisson process with rate $h$. As illustrated in Fig. 3(a), the response functions for different coupling $p_{\lambda}$ grow with external driving rate $h$ and saturate at a maximum firing rate $F_{\max }=\frac{1}{2+p_{\gamma}^{-1}} \mathrm{~ms}^{-1}$, which is determined by the refractory period $p_{\gamma}$. Among the response functions, there are three regimes. For very low coupling, the response functions are subcritical, the self-sustained solution is not allowed, and the activity dies out when $h \rightarrow 0$. On the high coupling limit, small perturbations lead the system to the self-sustained mode. In between both regimes there is the bistable region. Response functions in this regime are history dependent. Very small perturbations are typically not enough to drive the system to the self-sustained mode. However, at a certain external driving rate (which is trial dependent) the system becomes active, as depicted by the upward arrow in Fig. 3(a). On the contrary, if the response function is calculated by reducing the external driving (leftward arrow), the system maintains a high firing rate and the activity does not die out when $h \rightarrow 0$. This path dependence could explain the large fluctuations in the experimental response functions as well as the dependence on the measurement time period in the olfactory system [23].

The bistable regime also confers path dependence to the dynamic range. Figure 3(a) depicts the key elements of the standard dynamic range definition. The two horizontal dashed lines stand for $F_{0.1}$ (bottom) and $F_{0.9}$ (top). They correspond to $10 \%$ and $90 \%$ of the maximum firing rate $\left(F_{\max }\right)$ subtracted from the minimum firing rate $[F(h \rightarrow 0)]$, and they cross the response functions respectively at the external driving intensities of $h_{0.1}$ and $h_{0.9}$. The dynamic range is thus defined as the number of decades comprised between $h_{0.1}$ and $h_{0.9}$ : $\Delta \equiv 10 \log \frac{h_{0.9}}{h_{01}}$. Figures 3(b) and 3(d) show the dynamic range for networks of integrators with different integration times $\tau$, for random and scale-free networks. In the bistable regime, when a high firing rate is observed (bottom line), the system is only able to distinguish the input level intensity. For a low firing rate (top line), the system not only distinguishes the input intensity but also detects the abrupt change in the firing rate. The system displays the largest dynamic range in the low firing rate and the maximum appears in the bistable regime. The height and width of the peak of the dynamic range curves depend on the integration time. Coincidence detectors show a poor capacity to distinguish the incoming input (lower peak and narrower width of $\Delta$ as a function of $p_{\lambda}$ ).
However, for large enough density of integrators, the dynamic range increases with longer integration times [see Fig. 3(c)], which increases the capacity to discriminate incoming inputs. Table I compares the dynamic range of the neuronal networks with and without integrators: The maximum enhancement of the dynamic range as a consequence of the collective behavior [i.e., $\Delta^{\max }-\Delta\left(p_{\lambda}=0\right)$ ] is over four times larger in the presence (than in the absence) of integration in both random and scale-free networks.

Mean-field approach. The mean-field approximation we present corresponds to the model version for coincidence detection $\left(\tau_{I}\right)$. For $K \gg \theta$, the mean-field map for the average firing rate $F$ of a population with threshold $\theta$ can be written as

$$
\delta_{t} F_{t+1}=Q_{t} p_{h}+Q_{t}\left(1-p_{h}\right) \Lambda_{t}^{\theta},
$$

where $\Lambda_{t}^{\theta}=\left[1-\left(1-p_{\lambda} \delta_{t} F_{t}\right)^{K}\right]^{\theta}$ is the probability of a quiescent node to become active in the next time step due to at least $\theta$ neighbor contributions within a single time step, $Q_{t}=1-\delta_{t} F_{t}-R_{t}$ is the probability of finding a site in the quiescent state, and $R_{t+1}=\delta_{t} F_{t}+\left(1-p_{\gamma}\right) R_{t}$ is the probability of finding a site in the refractory state. Iterating the map until convergence we get the solution of $F$ in the stationary configuration $(t \rightarrow \infty)$, which is used to compare with the simulations. The numerical solutions of Eq. (1) for various conditions are shown in Figs. 1 and 3. For the population of nonintegrators [Fig. 1(a)] we recover the Kinouchi-Copelli equation [2], which describes particularly well the behavior in random networks. In the presence of integration, the result captures qualitatively the behavior of the phase transitions [Fig. 1(b)], the response function, and the dynamic range [Fig. 3(b)]. A bifurcation analysis reveals some aspects of the phase transition as a function of the threshold $\theta$. In the absence of input $(h=0)$, as shown in Fig. 4 , for $\theta=1$ there is a transcritical bifurcation; for $\theta>1$ a saddle-node bifurcation and a stable fixed point at $F=0$ coexist.

TABLE I. Dynamic range (dB) for network size $N=5000$.

\begin{tabular}{lccccc}
\hline \hline \multirow{2}{*}{ Network } & \multicolumn{2}{c}{ Nonintegrators $(\theta=1)$} & \multicolumn{2}{c}{ Integrators $\left(\theta=2, \tau_{\infty}\right)$} \\
\cline { 2 - 5 } \cline { 5 - 6 } & $\Delta^{\max }-\Delta(0)$ & $\Delta^{\max }$ & & $\Delta^{\max }-\Delta(0)$ & $\Delta^{\max }$ \\
\hline Random & 10 & 26 & 41 & 57 \\
Scale-free & 7 & 23 & 32 & 48 \\
\hline \hline
\end{tabular}



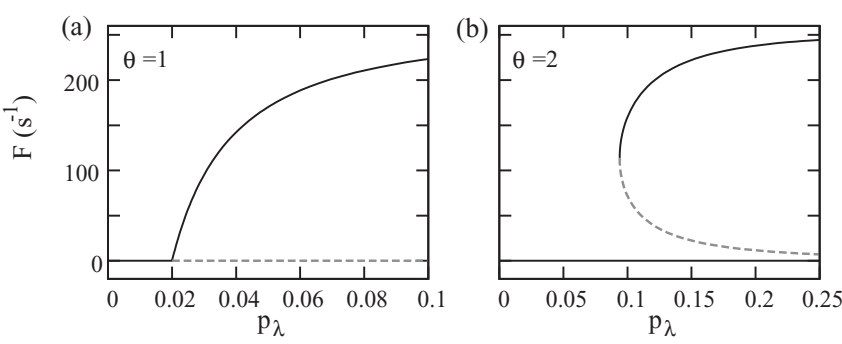

FIG. 4. Bifurcation diagram of the mean-field approximation: (a) transcritical for $\theta=1$; (b) saddle node for $\theta=2$. Solid lines, stable stationary solutions; dashed lines, unstable ones.

Analogously, one can also extend the results for heterogeneous populations, as considered in Fig. 2. At any time $t$, we define, for each sub-population of threshold $\theta_{i}, F_{t}^{\left(\theta_{i}\right)}$, $R_{t}^{\left(\theta_{i}\right)}$, and $Q_{t}^{\left(\theta_{i}\right)}$ as the firing rate, and the probability of finding a site in the refractory and in the quiescent states, respectively. The firing rate of the network is given by $F_{t}=F_{t}^{(1)}(1-d)+$ $F_{t}^{(2)} d$, where $d$ denotes the density of integrator nodes. Then, by generalizing Eq. (1) we can find $F^{(1)}$ and $F^{(2)}$ from:

$$
\delta_{t} F_{t+1}^{\left(\theta_{i}\right)}=Q_{t}^{\left(\theta_{i}\right)} p_{h}+Q_{t}^{\left(\theta_{i}\right)}\left(1-p_{h}\right) \Lambda_{t}^{\theta_{i}} .
$$

As shown in the left inset panel of Fig. 2, the average firing rate of the network qualitatively captures the phase transition.

Summary and conclusions. We have studied the collective behavior of an excitable media where the units integrate incoming signals [16]. The presence of a minimum density of integrator nodes leads the system to an abrupt phase transition. Discontinuous transitions have been observed experimentally and in threshold models [24], in models with adaptive interactions $[25,26]$, and in the presence of strong nonlinear coupling [27].
As a consequence of the discontinuous phase transition, bistability emerges. In the context of neuroscience, bistability is known to play an important role in memory maintenance [28]. A bistable regime composed of a configuration with high or low activity levels [29] has also been observed in cortical neurons. Since most neurons (if not all) must integrate their incoming postsynaptic potentials, our results suggest that the transition to the regime of self-sustained activity in a neuronal system could be restricted to a discontinuous transition type.

Concerning the output response to external stimulus (which might vary for orders of magnitude), the bistable regime provides two different response types, depending on the history (either with low or high activity levels for $h \sim 0$ ). The low past activity level with an infinite integration time gives rise to the largest dynamic range in random and scale-free networks. Taking this finding into account, biologically inspired artificial stimulus detectors with great capabilities can be designed from excitable media composed of integrator units [30]. Moreover, we expect that our results might also be relevant to other systems where integration plays an important role as, for instance, in gene regulatory networks [15], and social interaction [17], and it would be interesting to explore the behavior of the dynamic range in the recently found explosive percolation [31].

Acknowledgments. We thank to Ernest Motbrió, Miguel A. Muñoz, Ernesto M. Nicola, John Rinzel, and Olaf Sporns for useful discussions. L.L.G. acknowledges John Rinzel and his working group for hospitality and valuable discussions during his visit to the Center for Neural Science at New York University. The authors acknowledge financial support by grants from the MICINN (Spain) and FEDER under project FIS2007-60327 (FISICOS) and FIS2011-24785 (MODASS).
[1] V. M. Eguíluz, M. Ospeck, Y. Choe, A. J. Hudspeth, and M. O. Magnasco, Phys. Rev. Lett. 84, 5232 (2000); S. Camalet, T. Duke, F. Jülicher, and J. Prost, Proc. Natl. Acad. Sci. USA 97, 3183 (2000).

[2] O. Kinouchi and M. Copelli, Nat. Phys. 2, 348 (2006).

[3] W. Shew, H. Yang, T. Petermann, R. Roy, and D. Plenz, J. Neurosci. 29, 15595 (2009).

[4] J. M. Beggs and D. Plenz, J. Neurosci. 23, 11167 (2003).

[5] J. M. Beggs and D. Plenz, J. Neurosci. 24, 5216 (2004); J. M. Beggs, Philos. Trans. R. Soc. London A 366, 329 (2008); D. Plenz and T. C. Thiagarajan, Trends Neurosci. 30, 101 (2007); C. Haldeman and J. M. Beggs, Phys. Rev. Lett. 94, 058101 (2005); D. Hsu and J. Beggs, Neurocomputing 69, 1134 (2006).

[6] R. Legenstein and W. Maass, Neural Networks 20, 323 (2007).

[7] A. Levina, J. M. Herrmann, and T. Geisel, Nat. Phys. 3, 857 (2007).

[8] J. A. Bonachela, S. de Franciscis, J. J. Torres, and M. A. Muñoz, J. Stat. Mech.: Theory Exp. (2010) P02015.

[9] D. Fraiman, P. Balenzuela, J. Foss, and D. R. Chialvo, Phys. Rev. E 79, 061922 (2009).

[10] D. R. Chialvo, Physica A 340, 756 (2004); D. R. Chialvo, P. Balenzuela, D. Fraiman, L. M. Ricciardi, A. Buonocore, and E. Pirozzi, in Collective Dynamics: Topics on Competition and
Cooperation in the Biosciences: A Selection of Papers in the Proceedings of the BIOCOMP2007 International Conference, edited by L. M. Ricciardi, A. Buonocore, and E. Pirozzi, AIP Conf. Proc., Vol. 1028 (AIP, Melville, NY, 2008), p. 28; M. G. Kitzbichler, M. L. Smith, S. R. Christensen, and E. Bullmore, PLoS Comput. Biol. 5, e1000314 (2009); G. Werner, Front. Physiol. 1, 15 (2010); D. R. Chialvo, Nat. Phys. 6, 744 (2010); E. Tagliazucchi and D. R. Chialvo, e-print arXiv:1103.2070 (2011).

[11] L. L. Gollo, O. Kinouchi, and M. Copelli, Phys. Rev. E 85, 011911 (2012).

[12] L. L. Gollo, O. Kinouchi, and M. Copelli, PLoS Comput. Biol. 5, e1000402 (2009).

[13] L. S. Furtado and M. Copelli, Phys. Rev. E 73, 011907 (2006); A.-C. Wu, X.-J. Xu, and Y.-H. Wang, ibid. 75, 032901 (2007); M. Copelli and P. R. A. Campos, Eur. Phys. J. B 56, 273 (2007); V. R. V. Assis and M. Copelli, Phys. Rev. E 77, 011923 (2008).

[14] D. B. Larremore, W. L. Shew, and J. G. Restrepo, Phys. Rev. Lett. 106, 058101 (2011).

[15] A. Szejka, T. Mihaljev, and B. Drossel, New J. Phys. 10, 8 (2008).

[16] Essentials of Neural Science and Behavior, edited by E. R. Kandel, J. H. Schwartz, and T. M. Jessell (Appleton \& Lange, Norwalk, 1995). 
[17] P. S. Dodds and D. J. Watts, Phys. Rev. Lett. 92, 21870 (2004); M. S. Granovetter, Am. J. Sociol. 83, 1420 (1978); D. J. Watts, Proc. Natl. Acad. Sci. USA 99, 5766 (2002); D. Centola, V. M. Eguíluz, and M. W. Macy, Physica A 374, 449 (2007); D. Centola, Science 329, 1194 (2010).

[18] J. C. Magee, Nat. Rev. Neurosci. 1, 181 (2000).

[19] A. Reyes, Annu. Rev. Neurosci. 24, 653 (2001).

[20] N. Spruston, Nat. Rev. Neurosci. 9, 206 (2008).

[21] J. M. Greenberg and S. P. Hastings, SIAM J. Appl. Math. 34, 515 (1978).

[22] R. Albert and A.-L. Barabási, Rev. Mod. Phys. 74, 47 (2002).

[23] R. W. Friedrich and S. I. Korsching, Neuron 18, 737 (1997); M. Wachowiak and L. B. Cohen, ibid. 32, 723 (2001); V. Bhandawat, S. R. Olsen, N. W. Gouwens, M. L. Schlief, and R. I. Wilson, Nat. Neurosci. 10, 1474 (2007).
[24] I. Breskin, J. Soriano, E. Moses, and T. Tlusty, Phys. Rev. Lett. 97, 188102 (2006); O. Cohen, A. Keselman, E. Moses, M. R. Martínez, J. Soriano, and T. Tlusty, Europhys. Lett. 89, 18008 (2010).

[25] A. Levina, J. M. Herrmann, and T. Geisel, Phys. Rev. Lett. 102, 118110 (2009).

[26] D. Millman, S. Mihalas, A. Kirkwood, and E. Niebur, Nat. Phys. 6, 801 (2010).

[27] V. R. V. Assis and M. Copelli, Phys. Rev. E 80, 061105 (2009).

[28] J. M. Fuster and G. E. Alexander, Science 173, 652 (1971).

[29] M. Steriade, A. Nuñez, and F. Amzica, J. Neurosci. 13, 3252 (1993).

[30] B. N. S. Medeiros, V. Minces, G. B. Mindlin, M. Copelli, and J. R. Rios Leite, e-print arXiv:1110.0373 (2011). International Journal of Bifurcation and Chaos (in press).

[31] D. Achlioptas, R. M. D'Souza, and J. Spencer, Science 323, 1453 (2009). 